\title{
Performance Analysis of the Two Soliton Interactions in O-IDMA channel
}

\author{
Vimal Prakash Gautam ${ }^{1}$, Kapil Dev ${ }^{2}$, S.K. Sriwas ${ }^{3}$ \\ P.G. Student, Department of Electronics and Communication Engineering, B.I.E.T Jhansi, U.P, India ${ }^{1,2}$ \\ Assistant Professor, Department of Electronics and Communication Engineering, B.I.E.T Jhansi, U.P, India ${ }^{3}$
}

\begin{abstract}
The objective of the paper is to numerical computation of the two Soliton interaction in the optical interleave division multiple access channel. Solitons are used in O-IDMA because they have tendency to maintain their shape over a long distance. This occurred because of the interaction between nonlinearity and group velocity dispersion in anomalous region. Due to the individual pulse with, loss in O-IDMA channel and spacing between pulse, Copropagation Solitons do interact and share energy. Conduct various simulation. The interaction of two Slotion can be express with the help of Korteweg de Vries Equcation. Simulation is done with matlab. The simulation result shows that, two Soliton are travelling in channel and after a certain distance get attached and evolve a giant pulse. If propagation further continue then they get separated as if they walked through each other. With the help of simulation result, a careful choice of pulse, pulse separation and fiber parameter can be helpful for avoiding the Soliton interaction in O-IDMA channel. To improve the intensity of the Soliton prism mechanism can be used in O-IDMA channel.
\end{abstract}

Keywords: Soliton interaction, kerr effect, GVD, Nonlinearity.

\section{INTRODUCTION}

In the present world of wired communication, the optical fiber is generally used for attaining high spectral efficiency. These days, CDMA scheme is generally used in optical fiber based communication system. With the help of CDMA scheme, it can use proper bandwidth.

The CDMA scheme has been the popular for achieving better bandwidth efficiency than Frequency-Division MultipleAccess (FDMA) and Time-Division Multiple-Access (TDMA) schemes. The performance of Optical-CDMA (OCDMA) scheme also has some drawbacks i.e. multiple access interference (MAI) and inter-symbol interference (ISI). CDMA solution can be explained by another mechanism known as Interleave-Division Multiple-Access (IDMA). In IDMA we used different type of interleavers for user separation as per requirement.

The increased bandwidth demand has obsessed attention of the researchers to discover new path to streamline the quenched nits in the bandwidth pipe line. Dispersion, nonlinearity and the fiber losses are the limiting factor to design optical interleave multiple access communication system. Soliton is very useful in O-IDMA system, because it use the group velocity dispersion in anomalous region to counteract nonlinear self phase modulation. In other words, the nonlinearities is balanced by group velocity dispersion. Pulses are closely spaced so it required increased channel bandwidth. So, it is necessary to check into thoroughly interaction between co-propagating Soliton. In this work , various simulation have been performed using Matlab to investigate the interaction involving two Soliton.

\section{MATHEMATICAL MODELING FOR SOLITON INTERACTION}

The equation which propagate Soliton is the Non-linear Schrodinger equation (NLSE) for an optical pulse with the field envelope $u(z, t)$ propagating in the O-IDMA channel with no loss [1]

$\frac{\partial u}{\partial z}=\frac{-j}{2} \beta_{2} \frac{\partial^{2} u}{\partial t^{2}}+|u|^{2} u$

Where $\beta_{2}$ is the second order dispersion parameter. By solving the equation numerically with the amplitude consist of a Soliton pair the outcome of interaction on Soliton.

The Korteweg-deVries(KdV) equation, [2]

$\mathrm{u}_{\mathrm{t}}+\mathrm{uux}+\mathrm{u}_{\mathrm{xxx}}=0$

The KdV equation for Solitary wave:

$u(x, t)=3 \operatorname{csech}^{2} \frac{1}{2} \sqrt{c}(x-c t)$

The soliraty wave of the $\mathrm{KdV}$ equation shows that amplitude is directly proportional to the speed. 
The KdV equation special solution for solitary wave [3]

$\mathrm{u}(\mathrm{x}, \mathrm{t})=12 \omega^{2} \operatorname{sech}^{2}\left[\omega\left(\mathrm{x}-4 \omega^{2} \mathrm{t}\right)\right]$

These wave move right with speed $4 \omega^{2}$. There amplitude depends on the wave speed so the larger wave travel faster.

\section{SIMULATION RESULTS}

In this section, it shows the work with multiple sech field to analyze Soliton interactions in the O-IDMA. The two Soliton are collide in linear which is air and nonlinear medium which is O-IDMA channel.
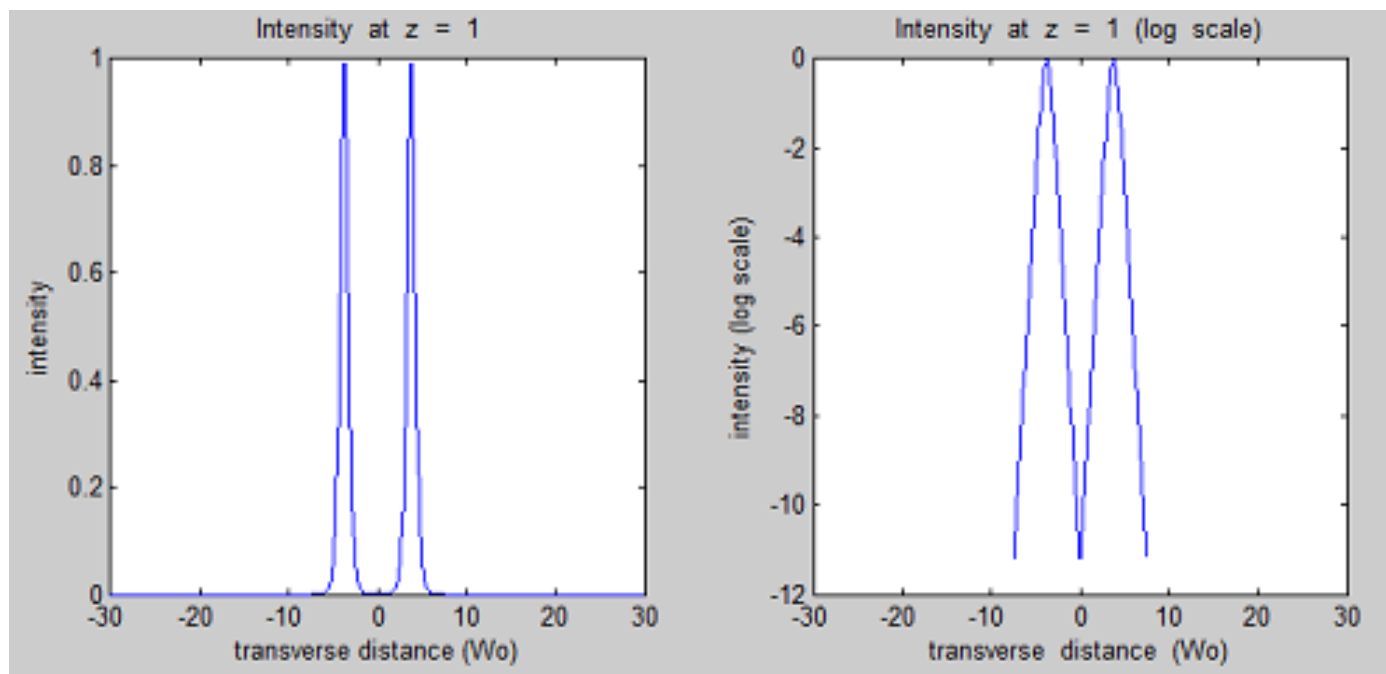

Figure 1: collision of two Soliton pulse in linear and linear medium at $\mathrm{Z}=1$

The simulation of collision of two Soliton in a linear medium Interference can be seen. The intensity image clearly shows that the Solitons remain unchanged after collision. Figure 2 shows the amplitude become null when the two Soliton are collide. There is phase discontinuity. The phase plot shows that intensity increase in near overlap region. It is shown in the 3D intensity plot.

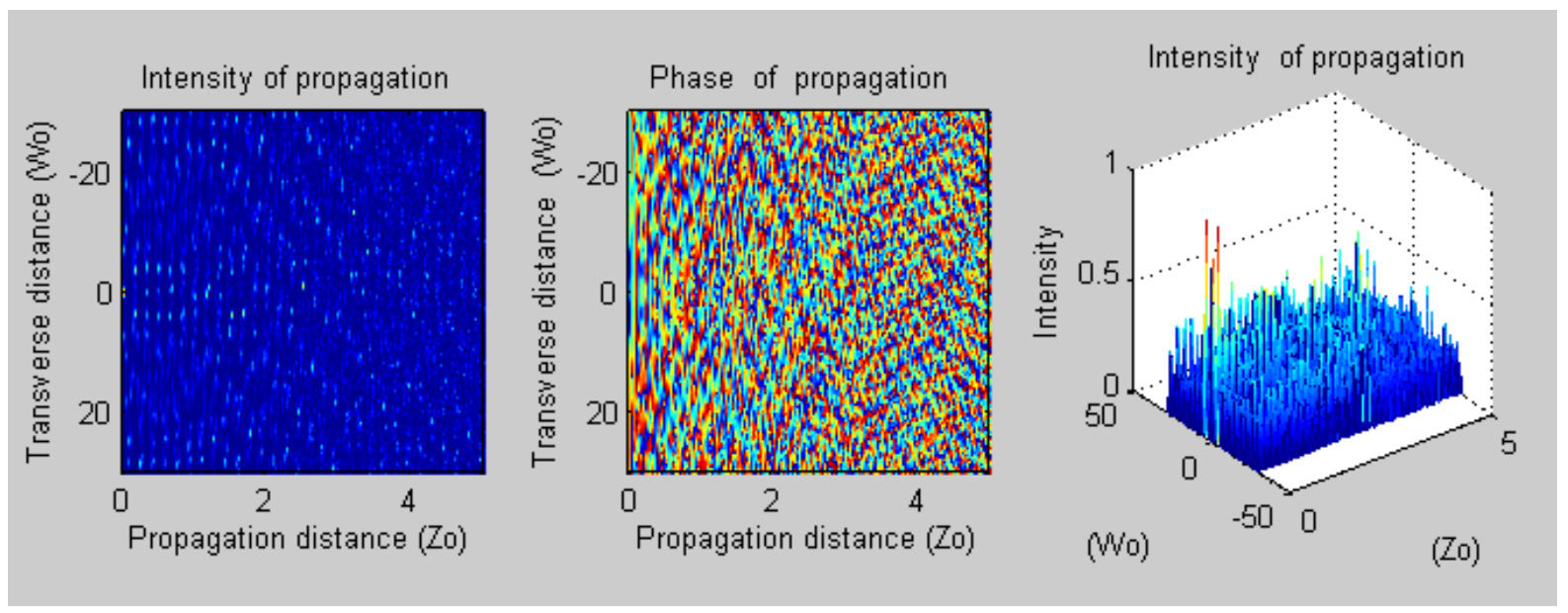

Figure2: intensity spectrum in linear medium

Soliton pulse propagation in the nonlinear medium shown in figure[3,4,5,6]. The simulation result shows that, two Soliton are travelling in channel and after a certain distance get attached and evolve a giant pulse. If propagation further continue then they get separated as if they walked through each other.

With the amplitude decreases, the spread of the wave increases . the solitary wave decreases exponential from their maximum. Slower waves have smaller rate. So one set the Solitons on a larger interval and integrate over a longer rate. This results in longer and longer computation times as the amplitude diminishes. Spreading of wave is due to nonlinear effect. 


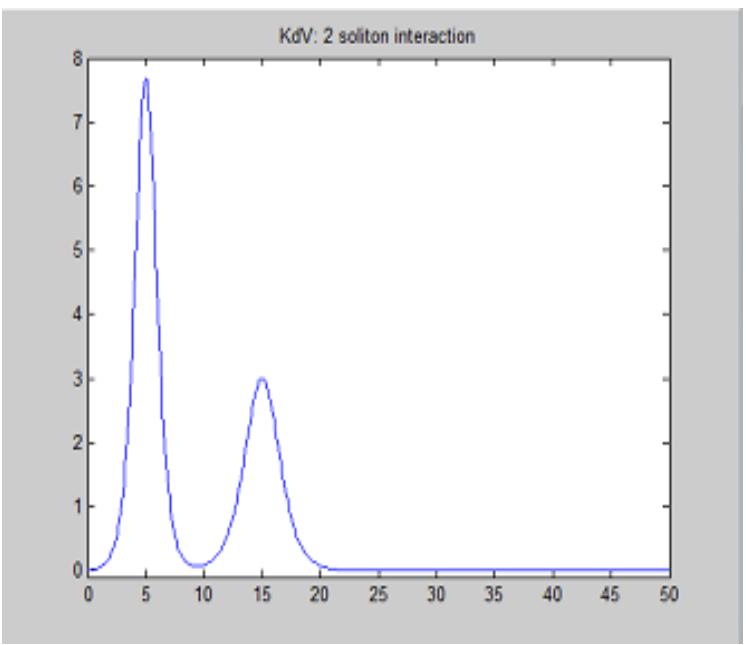

Figure3: two Solitons propagation at $\mathrm{t}=0$

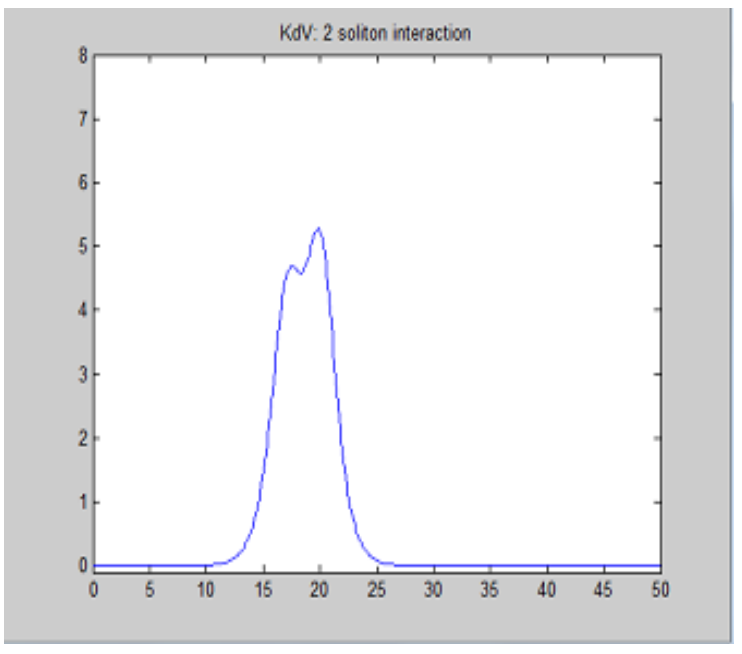

Figure5: two Solitons propagation at $\mathrm{t}=5$
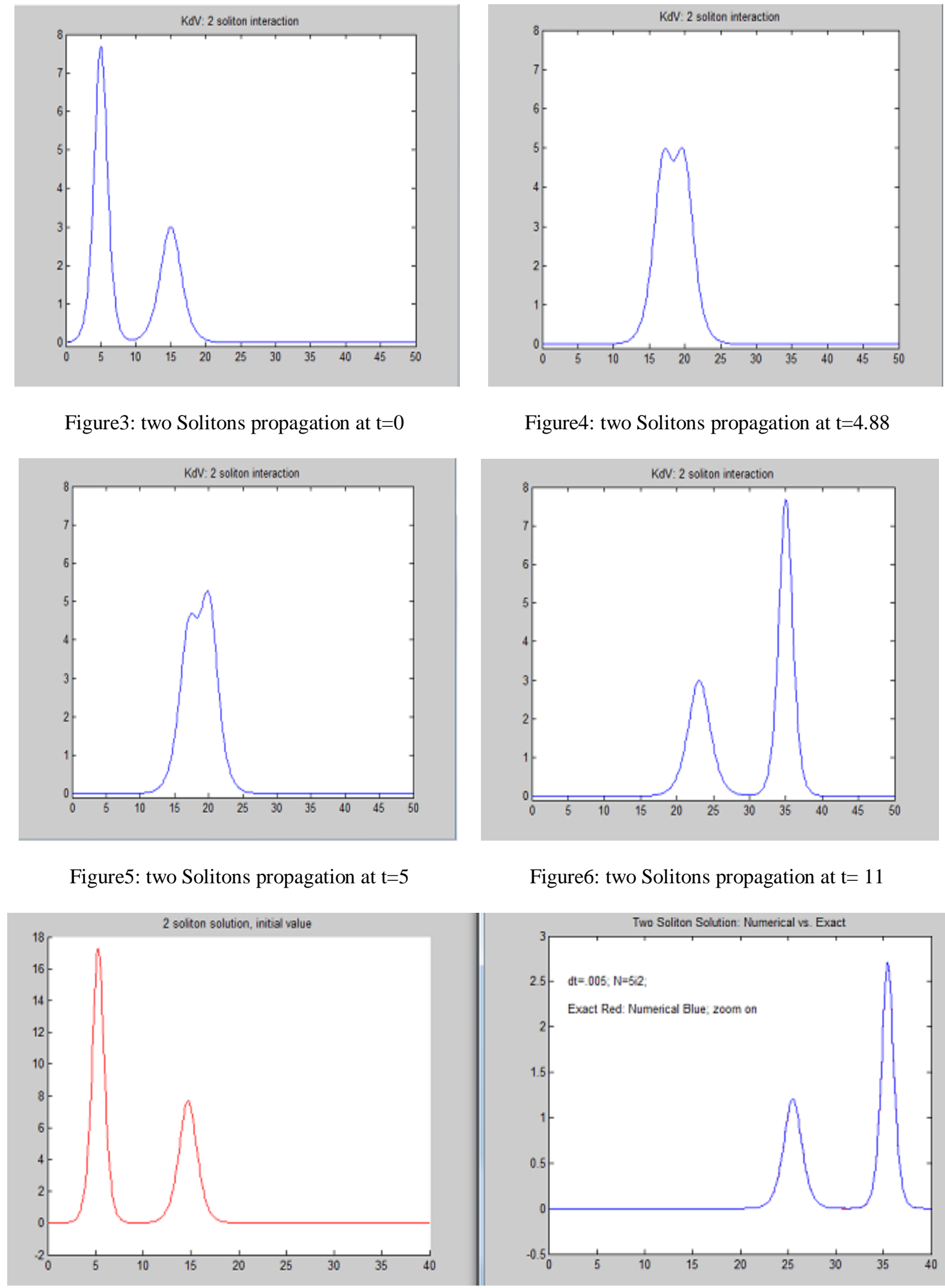

Figure4: two Solitons propagation at $\mathrm{t}=4.88$

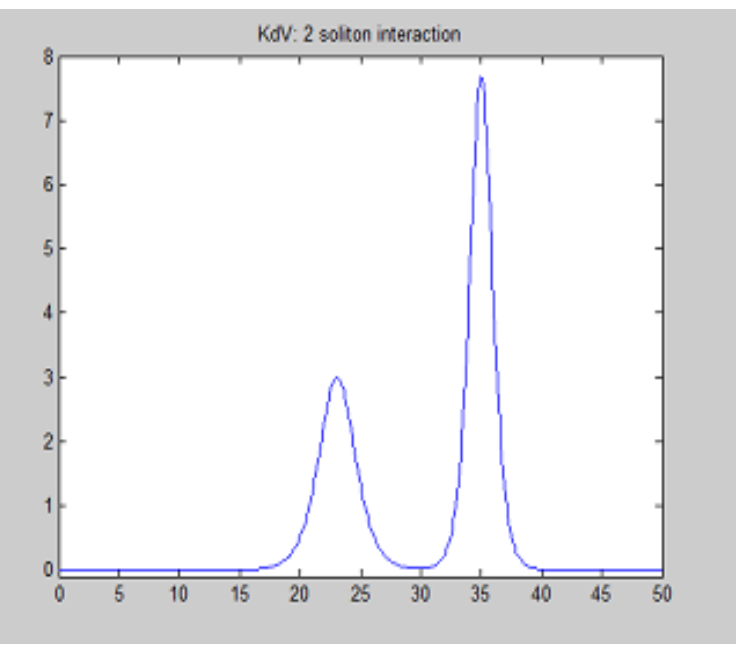

Figure6: two Solitons propagation at $\mathrm{t}=11$

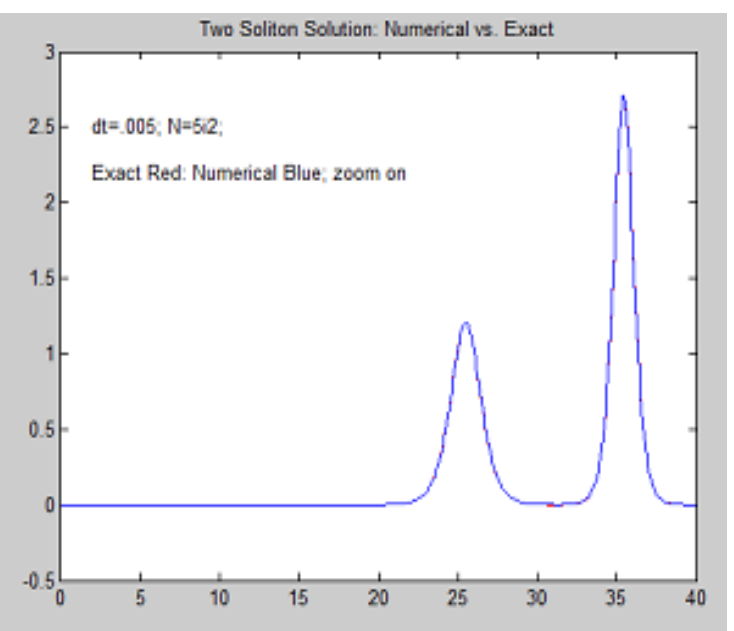

Figure7: Numerical computation of two Soliton pulse propagation

When Soliton passes through the nonlinear medium such as prism then with the help of intensity figure it shows the deflected beam intensity is greater than the incoming beam. It clearly shows that the deflected beam width reduced. 

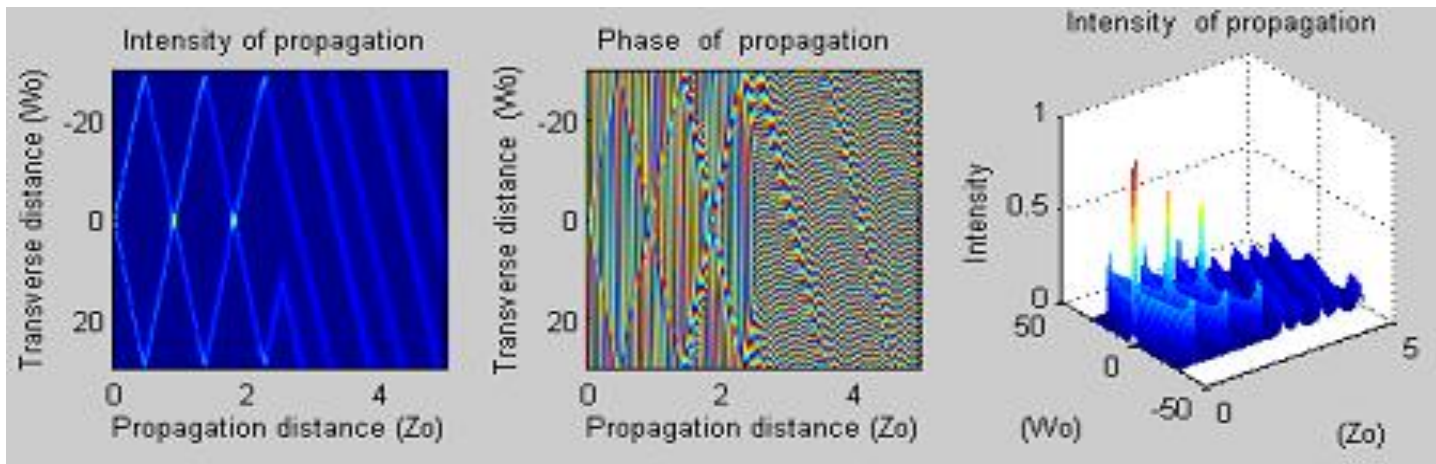

Figure 5: intensity spectrum through prism

\section{CONCLUSION}

Simulation analyzed the basic principle of optical Solitons and their interactions. Simulation analyzed the propagation of Soliton in linear and nonlinear medium. Comparison of the Soliton propagation in linear and nonlinear medium, it shows that peak intensity of the deflected beam is high as compared to incident beam. In this paper, it simulate the Soliton properties which is the amplitude depends upon the speed, phase modulation after nonlinear collision and the amplitude modulation during the nonlinear collision. Some difficulty arise to detect the Soliton signal because of the estimating parameter of the Soliton. Difficulty arise because the waveform shape varies as a function of unknown parameter. So use the multiple hypothesis for each parameter. the result shows the many interesting aspect of nonlinear dynamical system. Many vision were progressed into the behaviour of Solitons, as well as into an application of Soliton in optical IDMA channel .

\section{REFERENCES}

[1]. G.P. Agrawal, "Nonlinear Fiber Optics", San Diego, C A, Academic Press, Inc. $3^{\text {rd }}$ Edition, 2001.

[2]. Kruskal, M. "Nonlinear wave equation “, In J. Moser, editor, Dynamical Systems, Theory and Applications, volume 38,pp. 310-354, 1975.

[3]. Kruskal, M.D., \& Z.J. Zabusky. "Interaction of Solitons in a collisionless plasma and the recurrence of initial stages ", Phys. Rev. Lett, 240243,1965 .

[4]. Korteweg, D.J. \& de Vries, G. "On the change of form of long wavers advancing in a rectangular canal and on a new type of long stationary wave", Phil. Mag., pp. 39:422-443, 1895.

[5]. Keiser G. "Optical fiber Communications", McGraw-Hill Educations, $3^{\text {rd }}$ Edition, Singapore, 2000.

[6]. Weideman JAC and Herbst BM, "Split-Step methods for the solution of the nonlinear Schrodinger equation", SIAM Journal on Numerical Analysis, pp.485-507, 1986.

[7]. Iannone E, Matera F, Mecozzi A, Settlembre H. "Non linear optical communication network". Wiley, 1 stedition, New York USA, 1998.

[8]. Hasgawa, A., and Tappert, F, “Transmission of stationary nonlinear optical pulses in dispersive dielectric fibers', Appl. Phys. Lett, pp. 142-144, 1973.

[9]. P.K. Wai, C.R. Meyuk, Y. C. Lee, and H.H chen, "Nonlinear pulse propagation in the neighbourhood of the zero-dispersion wavelength of monomode optical fiber" Opt. Lett., pp.464-466, 1946.

[10]. P.K. Wai, C.R. Meyuk, Y. C. Lee, and H.H chen, "Nonlinear pulse propagation in the neighbourhood of the zero-dispersion wavelength of monomode optical fiber" Opt. Lett., pp.464-466, 1946.[11]. Prof. Kelvin Wagner-Fall, "Nonlinear/crystal optics", 2006 